\title{
Risk for Clostridioides difficile Infection among Older Adults with Cancer
}

\author{
Mini Kamboj, Renee L. Gennarelli, Jennifer Brite, Kent Sepkowitz, Allison Lipitz-Snyderman
}

\section{$\underset{\text { MDUCATION }}{\text { Medsce ACTIVITY }}$}

In support of improving patient care, this activity has been planned and implemented by Medscape, LLC and Emerging Infectious Diseases. Medscape, LLC is jointly accredited by the Accreditation Council for Continuing Medical Education (ACCME), the Accreditation Council for Pharmacy Education (ACPE), and the American Nurses Credentialing Center (ANCC), to provide continuing education for the healthcare team.

Medscape, LLC designates this Journal-based CME activity for a maximum of 1.00 AMA PRA Category 1 Credit(s) ${ }^{\mathrm{TM}}$. Physicians should claim only the credit commensurate with the extent of their participation in the activity.

Successful completion of this CME activity, which includes participation in the evaluation component, enables the participant to earn up to $1.0 \mathrm{MOC}$ points in the American Board of Internal Medicine's (ABIM) Maintenance of Certification (MOC) program. Participants will earn MOC points equivalent to the amount of CME credits claimed for the activity. It is the $\mathrm{CME}$ activity provider's responsibility to submit participant completion information to ACCME for the purpose of granting ABIM MOC credit.

All other clinicians completing this activity will be issued a certificate of participation. To participate in this journal CME activity: (1) review the learning objectives and author disclosures; (2) study the education content; (3) take the post-test with a $75 \%$ minimum passing score and complete the evaluation at http://www.medscape.org/journal/eid; and (4) view/print certificate. For CME questions, see page 1790.

Release date: August 21, 2019; Expiration date: August 21, 2020

Learning Objectives

Upon completion of this activity, participants will be able to:

- Describe findings of factors affecting risk for Clostridioides difficile infection (CDI) in older adults, according to a retrospective cohort study analysis using population-based SEER-Medicare data for 2011

- Determine findings of factors affecting risk for CDI in older adults, according to a nested case-control study analysis using population-based SEER-Medicare data for 2011

- Identify clinical implications of risk factors for CDI in older adults, according to a retrospective cohort study with a nested case-control analysis using population-based SEER-Medicare data for 2011

\section{CME Editor}

P. Lynne Stockton Taylor, VMD, MS, ELS(D), Technical Writer/Editor, Emerging Infectious Diseases. Disclosure: P. Lynne Stockton Taylor, VMD, MS, ELS(D), has disclosed no relevant financial relationships.

\section{CME Author}

Laurie Barclay, MD, freelance writer and reviewer, Medscape, LLC. Disclosure: Laurie Barclay, MD, has disclosed no relevant financial relationships.

\section{Authors}

Disclosure: Mini Kamboj, MD; Renee Lynn Gennarelli, MS; Jennifer Brite, DrPH; Kent Sepkowitz, MD; and Allison Lipitz-Snyderman, PhD, have disclosed no relevant financial relationships.

Author affiliations: Memorial Sloan Kettering Cancer Center, New York, New York, USA (M. Kamboj, R.L. Gennarelli, J. Brite, K. Sepkowitz, A. Lipitz-Snyderman); Weill Cornell Medical College, New York (M. Kamboj, K. Sepkowitz)

DOI: https://doi.org/10.3201/eid2509.181142
To assess whether risk for Clostridioides difficile infection (CDI) is higher among older adults with cancer, we conducted a retrospective cohort study with a nested case-control analysis using population-based Surveillance, Epidemiology, and End Results-Medicare linked data for 2011. Among 93,566 Medicare beneficiaries, incident CDI and odds for 
acquiring CDI were higher among patients with than without cancer. Specifically, risk was significantly higher for those who had liquid tumors and higher for those who had recently diagnosed solid tumors and distant metastasis. These findings were independent of prior healthcare-associated exposure. This population-based assessment can be used to identify targets for prevention of CDI.

$\mathrm{H}^{2}$ ealthcare-associated infections are common and often preventable infections that lead to high morbidity and mortality rates (1). The bacterium Clostridioides difficile (formerly Clostridium difficile) causes inflammation of the colon, which commonly causes diarrhea. In the United States, $C$. difficile infection (CDI) is the leading cause of healthcare-associated infections (2).

Among the general population, the risk for CDI among older adults ( $\geq 65$ years of age) is up to 26 times greater than that among younger adults (2). The effects of this infection are also worse for older persons. In 2015, CDI ranked as the 18th leading cause of death for persons $\geq 65$ years of age (3). Although persons in this age group accounted for only $48 \%$ of CDI cases, they accounted for $\approx 87 \%$ of deaths from CDI $(3,4)$.

For older adults, the risk for CDI may be amplified even further by cancer or its treatment. Common factors that influence risk for CDI in general are advanced age, diminished humoral immune response, healthcare-associated exposures, and extended receipt of antimicrobial drugs, all of which are widely prevalent aspects of oncologic and supportive care (5). In addition, exposure to certain cytotoxic chemotherapeutic drugs has been postulated to induce a loss of resistance to colonization with $C$. difficile, although the mechanisms are unclear $(6,7)$. Yet, the complex relationship between cancer in older adults and CDI remains weakly characterized at the population level $(8,9)$.

Our objective with this study was to determine whether the risk for CDI is higher among older adults with cancer than among older adults without cancer. A population-level assessment of cancer-specific incidence of CDI among older adults would not only increase our knowledge of the disease epidemiology in this specialized population but could also guide primary prevention strategies, focusing on those at greatest risk for development of this complication.

\section{Methods}

\section{Study Design}

This study was considered exempt research by the institutional review board of Memorial Sloan Kettering Cancer Center (New York, New York, USA). Use of Surveillance, Epidemiology, and End Results (SEER)-Medicare data for this study was approved by the National Cancer Institute (Bethesda, MD, USA).
We conducted a retrospective cohort study with a nested case-control analysis and used population-based SEERMedicare-linked data to assess CDI occurrence in 2011. The National Cancer Institute-sponsored SEER program includes regions that cover $\approx 28 \%$ of the US population. SEER registries include information about cancer site, initial treatments, and active follow-up for death. The SEERMedicare dataset links cancer registry files with Medicare enrollment information and claims for Medicare beneficiaries with a diagnosis of cancer. Compared with the US older adult population, the SEER-Medicare cohort has a similar age and sex distribution, slightly higher proportion of patients residing in urban and high-income areas, and smaller proportion of nonwhite persons (10). We used the random 5\% sample of Medicare beneficiaries who reside in SEER regions to identify non-cancer patients.

\section{Study Participants}

We included Medicare beneficiaries with and without a cancer diagnosis. For beneficiaries with cancer, we included those with solid (breast, colon, lung, prostate, and head and neck cancers) and liquid (lymphoma, myeloma, leukemia) tumors diagnosed during 2006-2010. We required that participants had been $\geq 66$ years of age at the time of diagnosis to allow for 1 year of claims to assess prior healthcare use and CDI diagnoses. For inclusion in the non-cancer patient sample, we required that Medicare beneficiaries had been $\geq 66$ years of age at the start of 2011 with no history of cancer.

All patients included in the cohort had to have been hospitalized $\geq 1$ time in 2011. We excluded those for whom Medicare Parts A and B coverage during 2010-2011 was incomplete and those who had a diagnosis of CDI in 2010 (the year before our study year).

\section{Variables}

CDI diagnoses were based on code 008.45 of the International Classification of Diseases, 9th Revision, on an inpatient claim. Because of a lack of dates for diagnosis codes on inpatient claims, the admission date of the first claim with a CDI diagnosis was used as the CDI date. We examined the following patient characteristics: age, sex, race, geographic region, urban/rural location, hospitalizations from the prior year (within 12 months before the index date), and stays in a skilled nursing facility from the prior year (within 12 months before the index date). Cancer status was categorized as cancer versus no cancer, as solid versus liquid tumors, and by individual disease types. For cancer patients, stage at diagnosis was categorized according to the SEER historic staging variable: in situ, local/regional, or distant or unknown.

\section{Cohort Analysis}

We determined CDI incidence in 2011 for hospitalized patients with and without cancer. Incidence was reported 
as the percentage of the cohort in whom CDI developed during the study period. We also examined the overall proportion of patients with CDI and CDI incidence by patient characteristics.

\section{Nested Case-Control Analysis}

We included all patients with a diagnosis of CDI in 2011 in the nested case-control analysis; the index date was defined as the date of first CDI diagnosis in 2011. We randomly selected 5 control participants for each case-patient, matched by age ( \pm 1 year) at study start date (January 1 , 2011) and sex. The index date for each control was the date of CDI for the matched case-patient. Each matched control must have had follow-up through at least the case-patient's index date with no occurrence of CDI from study start date through index date. Case-patients could not be their own controls, but a case-patient was eligible to be a control for another case-patient if the criteria were met.

We compared the cancer status and other characteristics of the case-patients and controls. We used conditional logistic regression models for individually matched casepatients and controls to obtain the odds of CDI among those with a cancer diagnosis. We calculated adjusted and unadjusted odds ratios (ORs) for CDI by tumor type, stage at diagnosis, and year of diagnosis; the reference group was non-cancer patients. The stage-specific model was run for solid tumors with the following 3 SEER categories: in situ, locoregional, or distant versus no cancer (reference group). Odds of CDI for patients with liquid tumors were not reported because liquid tumors are staged as distant disease. Odds of CDI occurrence were also calculated by prior hospitalizations and prior stays at a skilled nursing facility. All conditional logistic regression models included as covariates patient race, geographic region, urban/rural location, cancer status, prior hospitalizations, and prior stays at a skilled nursing facility. For the case-control analysis, we used stays within 12 months before a patient's index date to define prior hospitalizations and prior skilled nursing facility stays.

To examine associations among cancer patients who were likely to actively receive treatment, we also repeated the analysis for patients whose cancer was diagnosed during 2009-2010 only and those without a cancer diagnosis. We used the same method to obtain new matched case-patients and controls for this analysis. We considered $\mathrm{p}<0.05$ to be statistically significant. All analyses were conducted by using SAS version 9.4 (SAS Institute Inc., https://www.sas.com).

\section{Results}

\section{Cohort Analysis}

For the 93,566 beneficiaries in the study cohort, $2.6 \%$ had CDI during the study period. In unadjusted analyses, a higher proportion of patients with cancer had CDI (2.8\%) than did patients without cancer $(2.4 \%)$. The proportion with CDI was higher among female patients and patients living in the Northeast and metropolitan areas. When we analyzed by 5 -year age intervals, we found an incremental increase in CDI risk, from $1.9 \%$ among patients $66-69$ years of age to $2.9 \%$ among patients $\geq 85$ years of age (Table 1 ).

\section{Nested Case-Control Analysis}

For the 2,421 case-patients with CDI, we identified 12,105 matched controls. A higher proportion of case-patients $(54 \%)$ than controls $(49 \%)$ had cancer. The distribution of race was similar among case-patients and controls, but distribution by geography differed slightly. Higher proportions of case-patients than controls had been hospitalized multiple times before or had stayed in a skilled nursing facility (Table 2).

The odds of CDI developing were higher among cancer patients than non-cancer patients (adjusted OR 1.15, $95 \%$ CI $1.04-1.26 ; p=0.005$ ). When cancer was subdivided into solid and liquid tumor types, having a solid tumor was not significantly associated with an increased risk for CDI compared with having no cancer diagnosis (adjusted OR $1.05,95 \%$ CI $0.95-1.16$ ); an underlying diagnosis of a liquid tumor was significantly associated with increased risk for CDI compared with no cancer diagnosis (adjusted OR 1.74, 95\% CI 1.48-2.06; $\mathrm{p}<0.001$ ). When we restricted the analysis to only patients who had received a cancer diagnosis within the past 2 years or no cancer diagnosis at

\begin{tabular}{lcc}
\hline \multicolumn{3}{l}{ Table 1. Clostridioides difficile infection, by cohort characteristics, } \\
in study of risk among older & Tdults with cancer, United States \\
\hline & & \% Patients with \\
Characteristic & 93,566 & C. difficile infection \\
\hline Total & & 2.6 \\
\hline Cancer status & \\
Cancer & 47,323 & 2.8 \\
No cancer & 46,243 & 2.4 \\
\hline Age, y & & \\
$66-69$ & 12,001 & 1.9 \\
$70-74$ & 20,691 & 2.4 \\
$75-79$ & 20,155 & 2.6 \\
$80-84$ & 18,651 & 2.8 \\
>85 & 22,068 & 2.9 \\
\hline Sex & & \\
M & 42,330 & 2.4 \\
F & 51,236 & 2.8 \\
\hline Race & & \\
White & 77,831 & 2.6 \\
Black & 8,885 & 2.6 \\
Other/unknown & 6,850 & 2.2 \\
\hline Urban/rural & & \\
Metropolitan & 76,808 & 2.8 \\
Not metropolitan & 16,703 & 1.8 \\
\hline US region & & 2.5 \\
West & 36,726 & 3.0 \\
Midwest & 12,086 & 2.0 \\
South & 24,980 & 3.3 \\
Northeast & 19,774 & \\
\hline & & \\
\hline
\end{tabular}


Table 2. Characteristics of case-patients and controls in study of Clostridioides difficile infection in older adults with cancer, United States

\begin{tabular}{|c|c|c|}
\hline Characteristic & $\begin{array}{c}\text { \% Total case- } \\
\text { patients, } \\
n=2,421\end{array}$ & $\begin{array}{c}\% \text { Total } \\
\text { controls, } \\
n=12,105\end{array}$ \\
\hline \multicolumn{3}{|l|}{ Cancer diagnosis } \\
\hline No cancer & 46 & 51 \\
\hline Solid tumor & 43 & 43 \\
\hline Liquid tumor & 12 & 7 \\
\hline \multicolumn{3}{|l|}{ Age, $y^{*}$} \\
\hline $66-69$ & 10 & 10 \\
\hline $70-74$ & 20 & 21 \\
\hline $75-79$ & 21 & 21 \\
\hline $80-84$ & 22 & 22 \\
\hline$\geq 85$ & 27 & 27 \\
\hline \multicolumn{3}{|l|}{$\operatorname{Sex}^{*}$} \\
\hline $\mathrm{M}$ & 41 & 41 \\
\hline $\mathrm{F}$ & 59 & 59 \\
\hline \multicolumn{3}{|l|}{ Race } \\
\hline White & 84 & 83 \\
\hline Black & 10 & 9 \\
\hline Other/unknown & 6 & 7 \\
\hline \multicolumn{3}{|l|}{ Urban/rural } \\
\hline Metropolitan & 87 & 83 \\
\hline Not metropolitan & 13 & 17 \\
\hline \multicolumn{3}{|l|}{ US region } \\
\hline West & 38 & 40 \\
\hline Midwest & 15 & 13 \\
\hline South & 20 & 26 \\
\hline Northeast & 27 & 21 \\
\hline \multicolumn{3}{|l|}{ Prior hospitalizations $\dagger$} \\
\hline 0 & 20 & 37 \\
\hline 1 & 18 & 32 \\
\hline 2 & 19 & 16 \\
\hline 3 & 12 & 6 \\
\hline 4 & 10 & 4 \\
\hline$\geq 5$ & 22 & 5 \\
\hline \multicolumn{3}{|c|}{ Prior skilled nursing facility stay† } \\
\hline No & 56 & 83 \\
\hline Yes & 44 & 18 \\
\hline
\end{tabular}

all, we found that both solid and liquid tumor types were significantly associated with increased odds of CDI compared with no cancer diagnosis (Tables 3,4). Regardless of a cancer diagnosis, $\geq 2$ prior hospitalizations or a prior stay at a skilled nursing facility were each associated with increased odds of CDI occurrence (Table 3).

Findings from our adjusted stage-specific model demonstrated that the odds of CDI were higher for patients with a solid tumor than for those with no cancer (Figure). Odds of CDI were also higher for patients with solid cancer at an unknown stage at diagnosis, although this finding probably depends on the constructs of the historic staging variable in SEER. Odds of CDI did not differ significantly for cancer patients with in situ or local/regionalized solid tumors at diagnosis, compared with non-cancer patients.

\section{Discussion}

The main findings of our study, derived from a population-based cohort of Medicare beneficiaries $>65$ years of age, indicate that the risk for CDI is greater for older adults undergoing treatment for cancer than for agematched controls. We show that much of this excess risk is associated with the type of underlying cancer and advanced disease and remains independent of prior healthcare-associated exposure from inpatient and skilled nursing facility stays. Risk was highest for patients with hematologic malignancies. In comparison, for solid tumor patients, odds of developing CDI were higher only for those with a recent cancer diagnosis and those with distant metastasis at diagnosis.

Our findings collectively expand knowledge of how cancer diagnosis affects CDI-associated illness among older adults. Reports of CDI in the absence of exposure to antimicrobial drugs has been well described for patients receiving cytotoxic chemotherapy (11), plausibly related to

Table 3. Odds ratios of infection occurrence, nested case-control analysis in study of Clostridioides difficile infection in older adults with cancer

\begin{tabular}{|c|c|c|c|c|}
\hline \multirow[b]{2}{*}{ Characteristic } & \multicolumn{4}{|c|}{ Primary analysis: cancer patients and non-cancer patients* } \\
\hline & Unadjusted OR $(95 \% \mathrm{Cl})$ & $p$ value & Adjusted OR $(95 \% \mathrm{Cl}) \dagger$ & $\mathrm{p}$ value \\
\hline \multicolumn{5}{|l|}{ Tumor type } \\
\hline None & 1.00 & & 1.00 & \\
\hline Solid & $1.13(1.03-1.24)$ & 0.01 & $1.05(0.95-1.16)$ & 0.32 \\
\hline Liquid & $1.94(1.67-2.26)$ & $<0.001$ & $1.74(1.48-2.06)$ & $<0.001$ \\
\hline \multicolumn{5}{|c|}{ Prior hospitalizations } \\
\hline 0 & 1.00 & & 1.00 & \\
\hline 1 & $1.11(0.96-1.28)$ & 0.15 & $1.07(0.93-1.23)$ & 0.37 \\
\hline 2 & $2.33(2.02-2.69)$ & $<0.001$ & $1.83(1.56-2.14)$ & $<0.001$ \\
\hline 3 & $3.55(2.99-4.20)$ & $<0.001$ & $2.68(2.22-3.23)$ & $<0.001$ \\
\hline 4 & $4.93(4.09-5.96)$ & $<0.001$ & $3.49(2.81-4.31)$ & $<0.001$ \\
\hline$\geq 5$ & $8.63(7.39-10.09)$ & $<0.001$ & $5.76(4.74-6.99)$ & $<0.001$ \\
\hline \multicolumn{5}{|c|}{ Prior skilled nursing facility stay } \\
\hline No & 1.00 & & 1.00 & \\
\hline Yes & $3.93(3.56-4.33)$ & $<0.001$ & $1.59(1.39-1.82)$ & $<0.001$ \\
\hline
\end{tabular}

*For cancer patients, diagnosis was made during 2006-2010. OR, odds ratio.

†Adjusted odds of $C$. difficile infection based on conditional logistic regression model. Adjusted for age, sex, race, geographic region, urban/rural location, prior hospitalizations, and prior skilled nursing facility stays. Separate models were conducted for all patients with a cancer diagnosed $2006-2010$ and noncancer patients, and for recently diagnosed cancer patients (diagnosis 2009-2010) and non-cancer patients. 
Table 4. Adjusted odds ratios of Clostridioides difficile infection occurrence, nested case-control analysis

\begin{tabular}{|c|c|c|c|c|}
\hline \multirow[b]{2}{*}{ Characteristic } & \multicolumn{4}{|c|}{ Subanalysis: recently diagnosed cancer patients and non-cancer patients* } \\
\hline & Unadjusted OR $(95 \% \mathrm{Cl})$ & $p$ value & Adjusted OR $(95 \% \mathrm{Cl}) \dagger$ & $\mathrm{p}$ value \\
\hline \multicolumn{5}{|l|}{ Tumor type } \\
\hline None & 1.00 & & 1.00 & \\
\hline Solid & $1.36(1.22-1.53)$ & 0.01 & $1.24(1.10-1.40)$ & $<0.001$ \\
\hline Liquid & $2.22(1.83-2.69)$ & $<0.001$ & $1.84(1.49-2.26)$ & $<0.001$ \\
\hline \multicolumn{5}{|c|}{ Prior hospitalizations } \\
\hline 0 & 1.00 & & 1.00 & \\
\hline 1 & $1.13(0.96-1.33)$ & 0.13 & $1.07(0.91-1.26)$ & 0.40 \\
\hline 2 & $2.28(1.94-2.68)$ & $<0.001$ & $1.73(1.44-2.08)$ & $<0.001$ \\
\hline 3 & $3.17(2.60-3.86)$ & $<0.001$ & $2.32(1.86-2.88)$ & $<0.001$ \\
\hline 4 & $4.31(3.48-5.33)$ & $<0.001$ & $2.92(2.29-3.72)$ & $<0.001$ \\
\hline$>5$ & $8.34(7.00-9.94)$ & $<0.001$ & $5.25(4.21-6.55)$ & $<0.001$ \\
\hline \multicolumn{5}{|c|}{ Prior skilled nursing facility stay } \\
\hline No & 1.00 & & 1.00 & \\
\hline Yes & $3.80(3.40-4.24)$ & $<0.001$ & $1.64(1.41-1.92)$ & $<0.001$ \\
\hline
\end{tabular}

*For cancer patients, diagnosis was made during 2009-2010. OR, odds ratio.

†Adjusted odds of CDI based on conditional logistic regression model. Adjusted for age, sex, race, geographic region, urban/rural location, prior

hospitalizations, and prior skilled nursing facility stays. Separate models were conducted with all patients with a cancer diagnosis during 2006-2010 and noncancer patients, and for recently diagnosed cancer patients (those diagnosed 2009-2010) and noncancer patients.

intestinal dysbiosis caused by these agents. In our study, the occurrence of CDI seemed to be influenced by active oncologic therapy among patients with solid tumors, as suggested by the heightened risk at the time of new diagnosis, when the intensity of treatment is expected to be greater. Similarly, the CDI risk for patients with solid tumors was also higher for those with distant site metastases at the time of diagnosis, underscoring the complexities in management of advanced cancers. We cannot determine from this study whether these associations are driven by type of cancer treatment, excessive use of antimicrobial drugs, or a consequence of immunologic and microbial perturbation associated with cancer treatment in persons of advanced age.
We were unable to quantify antecedent exposure to antimicrobial drugs, a major driver of CDI (12-15); the coexistence of several confounding factors makes this distinction particularly challenging. When we made risk adjustments for race, geographic region, type of healthcare facility, and number of prior healthcare facility stays, the odds of CDI in patients with liquid tumors were significantly higher. Similar to previous studies of non-cancer populations, we found an incremental increase in CDI risk with number of hospitalizations; risk for those with $>3$ hospital stays was 3 -fold higher $(12,16)$.

Symptoms of primary and recurrent CDI can be particularly debilitating in persons with advanced age and

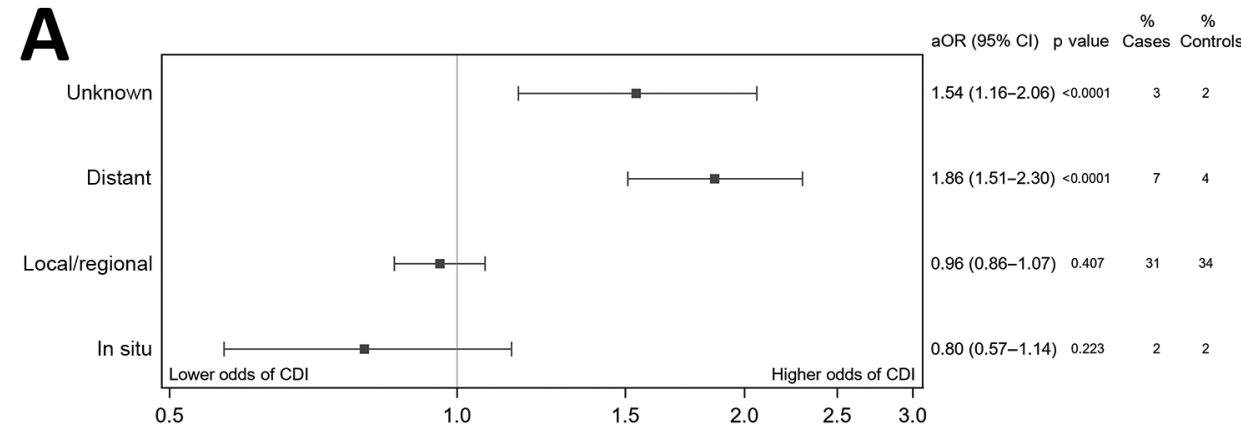

B

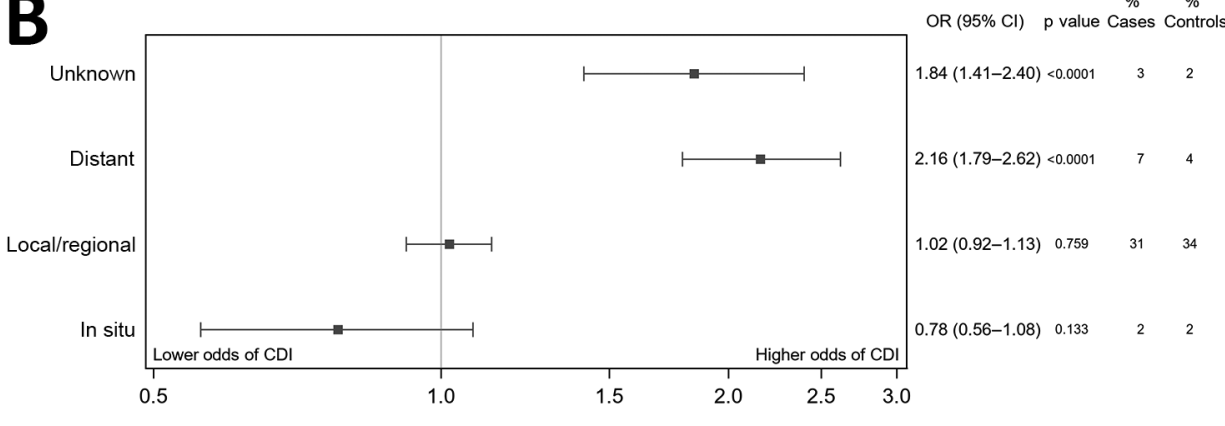

Figure. Adjusted $(A)$ and unadjusted $(B)$ odds of CDI for cancer patients with solid tumor compared with non-cancer patients. Stage is based on Surveillance, Epidemiology, and End Results historic staging variable. aOR estimates were generated from a logistic regression model adjusted for age, sex, race geographic region, urban/rural location, prior hospitalization, and prior skilled nursing stay. Non-cancer patients serve as the reference group, indicated by the reference line at 1.0. Error bars indicate 95\% Cls. aOR, adjusted odds ratio; CDI, Clostridioides difficile infection; OR, odds ratio. 
are frequently associated with postinfectious irritable bowel syndrome (5). In patients receiving concomitant cancer therapy, CDI symptoms are often indistinguishable from the gastrointestinal side effects of chemotherapy, radiation, and newer immunotherapies (17). For these complex reasons, CDI during cancer treatment can lead to delays in future chemotherapy or radiation cycles and have been shown to negatively affect eligibility for curative treatment options (18). In addition, the estimated surplus healthcare-associated costs of primary CDI in patients with advanced age is estimated to be around US $\$ 37,000$ and for patients with immunocompromising conditions US $\$ 16,000$, almost 2-fold higher than costs of CDI for patients without immunocompromising conditions (19). The wide-ranging effects of CDI in this population warrants assessment of primary prevention strategies (20-22). Our study defines the subset of older adults with cancer who would probably benefit the most from such therapies to minimize the vulnerability to CDI during cancer treatment.

The strength of our study is that it provides population-based quantitative estimates of the differential effects of CDI among older adults with and without cancer. Despite the many advantages of the SEER-Medicare dataset, our study has limitations: we were able to measure CDI-related disease burden only in the hospital setting, and community-acquired (or community-onset) cases were not included unless they resulted in a hospitalization. Although we excluded patients who received a CDI diagnosis in 2010 , case-patients do not necessarily represent those with primary infections. We used only the first Medicare claim with a CDI diagnosis; outpatient diagnoses would be missed and recurrent disease could potentially be represented as incident CDI. In addition, we did not measure relapsing CDI.

In summary, the burden of CDI among older adults is greater among those with underlying cancer. Regardless of prior healthcare-associated exposure, risk for CDI was highest for patients with hematologic malignancy and those with recent diagnosis of solid tumor or distant metastatic disease at diagnosis. These findings can be used to guide CDI prevention strategies.

Funding for this study was provided by the National Institutes of Health, National Cancer Institute Cancer Center Support Grant P30 (CA008748).

\section{About the Author}

Dr. Kamboj is an infectious disease physician and hospital epidemiologist at Memorial Sloan Kettering Cancer Center in New York, NY. Her research interests include the epidemiology of healthcare-associated infections in patients undergoing cancer treatment.

\section{References}

1. Magill SS, Edwards JR, Bamberg W, Beldavs ZG, Dumyati G, Kainer MA, et al.; Emerging Infections Program HealthcareAssociated Infections and Antimicrobial Use Prevalence Survey Team. Multistate point-prevalence survey of health care-associated infections. N Engl J Med. 2014;370:1198-208. https://doi.org/ 10.1056/NEJMoa1306801

2. Lessa FC, Mu Y, Bamberg WM, Beldavs ZG, Dumyati GK, Dunn JR, et al. Burden of Clostridium difficile infection in the United States. N Engl J Med. 2015;372:825-34. https://doi.org/ 10.1056/NEJMoa1408913

3. Murphy SL, Xu J, Kochanek KD, Curtin SC, Arias E. Deaths: final data for 2015. Natl Vital Stat Rep. 2017;66:1-75.

4. Centers for Disease Control and Prevention. 2015 Annual Report for the Emerging Infections Program for Clostridium difficile Infection [cited 2018 Jun 18]. https://www.cdc.gov/hai/eip/ Annual-CDI-Report-2015.html

5. Neemann K, Freifeld A. Clostridium difficile-associated diarrhea in the oncology patient. J Oncol Pract. 2017;13:25-30. https://doi.org/10.1200/JOP.2016.018614

6. Anand A, Glatt AE. Clostridium difficile infection associated with antineoplastic chemotherapy: a review. Clin Infect Dis. 1993;17:109-13. https://doi.org/10.1093/clinids/17.1.109

7. Loo VG, Bourgault AM, Poirier L, Lamothe F, Michaud S, Turgeon N, et al. Host and pathogen factors for Clostridium difficile infection and colonization. N Engl J Med. 2011;365: 1693-703. https://doi.org/10.1056/NEJMoa1012413

8. Chopra T, Alangaden GJ, Chandrasekar P. Clostridium difficile infection in cancer patients and hematopoietic stem cell transplant recipients. Expert Rev Anti Infect Ther. 2010;8:1113-9. https://doi.org/10.1586/eri.10.95

9. Kamboj M, Son C, Cantu S, Chemaly RF, Dickman J, Dubberke E, et al. Hospital-onset Clostridium difficile infection rates in persons with cancer or hematopoietic stem cell transplant: a C3IC network report. Infect Control Hosp Epidemiol. 2012;33:1162-5. https://doi.org/10.1086/668023

10. Warren JL, Klabunde CN, Schrag D, Bach PB, Riley GF. Overview of the SEER-Medicare data: content, research applications, and generalizability to the United States elderly population. Med Care. 2002;40(Suppl):IV-3-18. https://doi.org/10.1097/ 00005650-200208001-00002

11. Kamthan AG, Bruckner HW, Hirschman SZ, Agus SG. Clostridium difficile diarrhea induced by cancer chemotherapy. Arch Intern Med. 1992;152:1715-7. https://doi.org/10.1001/ archinte.1992.00400200139025

12. Cohen SH, Gerding DN, Johnson S, Kelly CP, Loo VG, McDonald LC, et al.; Society for Healthcare Epidemiology of America; Infectious Diseases Society of America. Clinical practice guidelines for Clostridium difficile infection in adults: 2010 update by the Society for Healthcare Epidemiology of America (SHEA) and the Infectious Diseases Society of America (IDSA). Infect Control Hosp Epidemiol. 2010;31:431-55. https://doi.org/ $10.1086 / 651706$

13. McFarland LV, Clarridge JE, Beneda HW, Raugi GJ. Fluoroquinolone use and risk factors for Clostridium difficileassociated disease within a Veterans Administration health care system. Clin Infect Dis. 2007;45:1141-51. https://doi.org/ $10.1086 / 522187$

14. Nelson DE, Auerbach SB, Baltch AL, Desjardin E, Beck-Sague C, Rheal C, et al. Epidemic Clostridium difficile-associated diarrhea: role of second- and third-generation cephalosporins. Infect Control Hosp Epidemiol. 1994;15:88-94. https://doi.org/10.2307/30145537

15. Stevens V, Dumyati G, Fine LS, Fisher SG, van Wijngaarden E. Cumulative antibiotic exposures over time and the risk of Clostridium difficile infection. Clin Infect Dis. 2011;53:42-8. https://doi.org/10.1093/cid/cir301 
16. Tabak YP, Johannes RS, Sun X, Nunez CM, McDonald LC. Predicting the risk for hospital-onset Clostridium difficile infection (HO-CDI) at the time of inpatient admission: HO-CDI risk score. Infect Control Hosp Epidemiol. 2015;36:695-701. https://doi.org/ 10.1017/ice.2015.37

17. Kamboj M, Brite J, Aslam A, Kennington J, Babady NE, Calfee D, et al. Artificial differences in Clostridium difficile infection rates associated with disparity in testing. Emerg Infect Dis. 2018;24:584-7. https://doi.org/10.3201/eid2403.170961

18. Hautmann MG, Hipp M, Kölbl O. Clostridium difficile-associated diarrhea in radiooncology: an underestimated problem for the feasibility of the radiooncological treatment? Radiat Oncol. 2011;6:89. https://doi.org/10.1186/1748-717X-6-89

19. Zhang D, Prabhu VS, Marcella SW. Attributable healthcare resource utilization and costs for patients with primary and recurrent Clostridium difficile infection in the United States. Clin Infect Dis. 2018;66:1326-32. https://doi.org/10.1093/cid/cix1021
20. Wilcox MH, Gerding DN, Poxton IR, Kelly C, Nathan R, Birch T, et al.; MODIFY I and MODIFY II Investigators. Bezlotoxumab for prevention of recurrent Clostridium difficile infection. N Engl J Med. 2017;376:305-17. https://doi.org/10.1056/ NEJMoa1602615

21. Lewis BB, Pamer EG. Microbiota-based therapies for Clostridium difficile and antibiotic-resistant enteric infections. Annu Rev Microbiol. 2017;71:157-78. https://doi.org/10.1146/ annurev-micro-090816-093549

22. Martin J, Wilcox M. New and emerging therapies for Clostridium difficile infection. Curr Opin Infect Dis. 2016;29:546-54. https://doi.org/10.1097/QCO.0000000000000320

Address for correspondence: Mini Kamboj, Memorial Sloane Kettering Cancer Center, 1275 York Ave, New York, NY 10065, USA; email: kambojm@mskcc.org

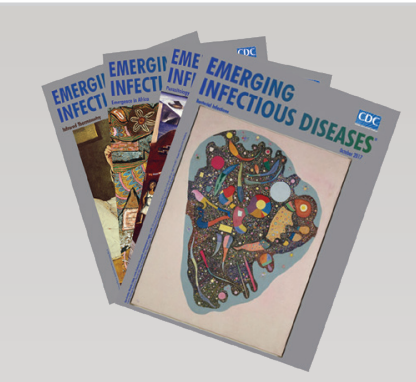

- Fatal Rocky Mountain Spotted Fever along the United States-Mexico Border, 2013-2016

- Surveillance of Extrapulmonary Nontuberculous Mycobacteria Infections, Oregon, USA, 2007-2012

- Investigation of Outbreaks of Salmonella enterica Serovar Typhimurium and Its Monophasic Variants Using Whole-Genome Sequencing, Denmark

- Enteric Infections Circulating during Hajj Seasons, 2011-2013

- Economic Assessment of Waterborne Outbreak of Cryptosporidiosis

- Antimicrobial Drug Prescription and Neisseria gonorrhoeae Susceptibility, United States, 2005-2013

- Poliovirus Excretion in Children with Primary Immunodeficiency Disorders, India

- Disease Burden of Clostridium difficile Infections in Adults, Hong Kong, China, 2006-2014

- Molecular Tracing to Find Source of Protracted Invasive Listeriosis Outbreak, Southern Germany, 2012-2016

- Dengue Virus 1 Outbreak in Buenos Aires, Argentina, 2016

- Mild Illness during Outbreak of Shiga Toxin-Producing Escherichia coli $\mathrm{O} 157$ Infections Associated with Agricultural Show, Australia

- Enterovirus D68-Associated Acute Flaccid Myelitis in Immunocompromised Woman, Italy

\section{October 2017}

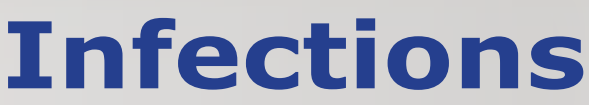

- Diagnosis of Fatal Human Case of St. Louis Encephalitis Virus Infection by Metagenomic Sequencing, California, 2016

- Usutu Virus RNA in Mosquitoes, Israel, 2014-2015

- Macrolide-Resistant Mycoplasma pneumoniae Infection, Japan, 2008-2015

- Epidemiology of Reemerging Scarlet Fever, Hong Kong, 2005-2015

- Off-Label Use of Bedaquiline in Children and Adolescents with Multidrug-Resistant Tuberculosis

- Monitoring Avian Influenza Viruses from Chicken Carcasses Sold at Markets, China, 2016

- Bedaquiline and Delamanid Combination Treatment of 5 Patients with Pulmonary Extensively Drug-Resistant Tuberculosis

- Hantavirus Pulmonary Syndrome Caused by Maripa Virus in French Guiana, 2008-2016

- Bedaquiline and Linezolid for Extensively Drug-Resistant Tuberculosis in Pregnant Woman

- Carbapenemase VCC-1-Producing Vibrio cholerae in Coastal Waters of Germany

- Autochthonous Transmission of East/Central/South African Genotype Chikungunya Virus, Brazil

- Fluoroquinolone-Resistant Alcaligenes faecalis Related to Chronic Suppurative Otitis Media, Angola 